\title{
Soft Tissue Measurement Method Using Radiopaque Material on Cone-beam Computed Tomography: An Ex Vivo Validation Study
}

\author{
Hae-Seok Lee', Jeong-Ho Yun², Dong-Won Lee ${ }^{1 *}$ \\ 'Department of Periodontology, Gangnam Severance Dental Hospital, College of Dentistry, Yonsei University, \\ Seoul, Korea \\ ${ }^{2}$ Department of Periodontology, School of Dentistry and Institute of Oral Bioscience, Chonbuk National \\ University, Jeonju, Korea \\ *Corresponding author: Dong-Won Lee, periodong@yuhs.ac
}

OPEN ACCESS

pISSN : 1229-5418

Implantology 2018; 22(4): 210-218

https://doi.org/10.32542/implantology.20180017

Received: November 27, 2018

Revised: December18, 2018

Accepted: December18, 2018

Copyright $($ 2018. The Korean Academy of Oral \& Maxillofacial Implantology

(c) (5) This is an Open Access article distributed under the terms of the Creative Commons Attribution Non-Commercial License (http://creativecommons. org/licenses/by-nc/4.0/) which permits unrestricted non-commercial use, distribution, and reproduction in any medium, provided the original work is properly cited.

\section{Abstract}

Purpose: The purpose of this study was to investigate the validity and reproducibility of a method based on cone-beam computed tomography (CBCT) technology for the visualization and measurement of gingival soft-tissue dimensions.

Material and Methods: A total of 66 selected points in soft-tissue of the ex vivo head of an adult pig were investigated in this study. For the measurement of radiographic thickness (RT), wet softtissue surfaces were lightly covered with barium sulfate powder using a powder spray. CBCT was taken and DICOM files were assessed for soft-tissue thickness measurement at reference points. A periodontal probe and a rubber stop were used for the measurement of trans-gingival probing thickness (TPT). After flap elevation, actual thickness of soft-tissue (actual thickness, AT) was measured. Correlation analysis and intraclass correlation coefficients analysis (ICC) were performed for AT, TPT, and RT.

Results: All variables were distributed normally. Strong significant correlations of AT with RT and TPT values were found. The two ICC values between TPT vs. AT and RT vs. AT differed significantly.

Conclusion: Our results indicated that correlation of RT was stronger than that of TPT with AT. We concluded that soft tissue measurement with $\mathrm{CBCT}$ could be a reliable method, compared to the trans-gingival probing measurement method.

Keywords: Cone-beam computed tomography, Dimensional Measurement Accuracy, Imaging

\section{I . Introduction}

Previously, a noninvasive method using a radiopaque material and periapical radiography to measure the vertical length of the interdental papilla in natural teeth and implants was proposed $^{1-3}$. By using radiopaque material, it was possible to demarcate soft tissue, without underexposing radiography. However, such two-dimensional information limits the assessment of the whole periodontium. 
The metric assessment of soft tissue dimensions around teeth and implants is of great clinical interest for the quantification and monitoring of gingival changes during therapies. Gingival soft tissue dimensions play significant roles in the assessment of whole treatment success, and thus should be monitored all through therapy. A lack of gingival thickness showed a tendency towards loss in attachment levels after traumatic, inflammatory, or surgical injuries ${ }^{4}$. Likewise, orthodontic tooth movement may adversely affect the mucogingival complex, especially at sites in which the keratinized gingiva and underlying bone appeared thin ${ }^{5}$. Acceptable methods for the accurate quantification of tissue changes when assessing new treatment modalities and materials influencing soft tissues are thus needed ${ }^{6}$.

Cone-beam computed tomography (CBCT) is used routinely for imaging analyses of the maxillofacial region $^{7}$. This modality provides clinicians with high-quality diagnostic images and has become an important tool in dentistry. However, the inability of CBCT to distinguish overlapped soft tissues, such as mucogingival thickness on the buccal side of alveolar bone, has limited its application exclusively to the imaging of hard maxillofacial tissues ${ }^{8}$.

Several studies reported using $\mathrm{CBCT}^{9-12}$ for studying soft tissue thickness. Although validation process was not reported, these studies showed us a possibility to study delicate mucogingival soft tissue by retracting overlapped soft tissue. However, one validation study reported that soft tissues less than $0.5 \mathrm{~mm}$ was not possible to be confirmed in spiral CT, thus making the application of spiral CT in very thin mucosa questionable ${ }^{13}$. In this study, we describe a method based on CBCT technology for the visualization and measurement of soft tissue dimensions, after demarcating the soft tissue with radiopaque material. The aim of this study was to investigate the validity and reproducibility of this method.

\section{Material and Methods}

For this study, the ex vivo head of an adult pig was used. The test sites were confined to the soft tissue around the posterior teeth. Radiographic markers made of radiopaque flowable composite (EsthetXflow A3; Dentsply, Milford, DE, USA) were applied to the enamel cusps of each tooth and light cured (EliparFreeLight 2; 3M ESPE, St. Paul, MN, USA). A total of 22 markers were obtained (Fig. 1). To align CBCT section and actual measurement as close as possible, CBCT cross section contained two composite markers. Actual measurement and trans gingival probing were performed on the imaginary line connecting two composite markers. For the measurement of soft tissue thickness, we chose 9, 12, and $15 \mathrm{~mm}$ apical to the flowable composite markers. Thus, a total of 66 selected points in soft tissue were investigated in this study. 


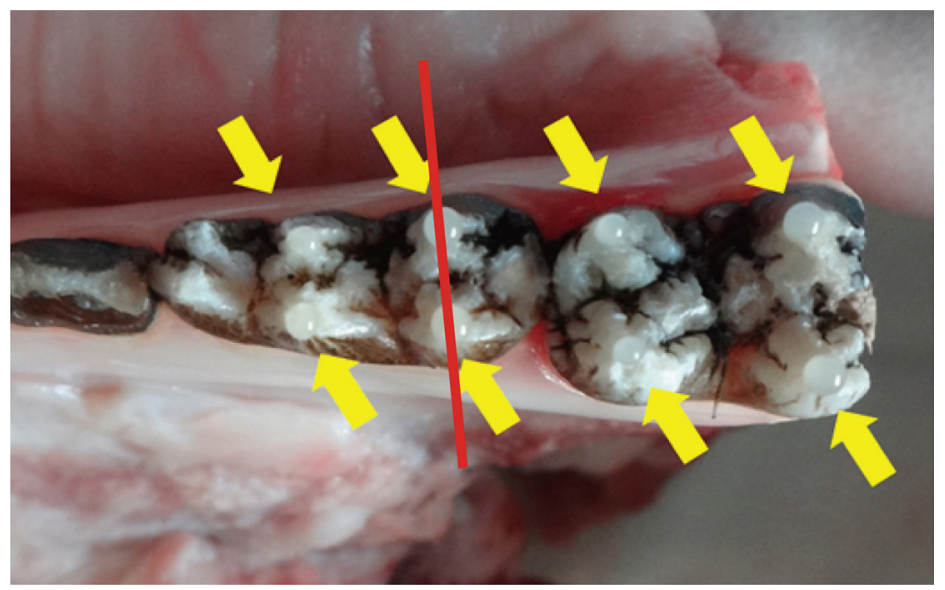

Fig. 1. Radiopaque flowable composite markers were attached to the enamel (yellow arrows). Red line indicates imaginary line connecting two composite markers. These line will be the CBCT cross section and AT and TPT will be performed.

Hae-Seok Lee et al. : Soft Tissue Measurement Method Using Radiopaque Material on Cone-beam Computed Tomography: An Ex Vivo Validation Study. Implantology 2018

\section{Testing intra-observer variability}

The measurements were done by a single operator. Prior to taking part in the present investigation, intra-observer variability was tested under the supervision of the director. 3 parameters were tested. Thus, 40 arbitrary selected sites on pig mandible were measured with 1) DICOM viewer (Simplant ${ }^{\circledR}$; Materialise NV, Leuven, Belgium) after CBCT (Voxel size 0.08 mm, Pax-Zenith 3D; Vatech, Seoul, Korea) taking, 2) trans-gingival probing and measuring with caliper, 3) actual soft tissue thickness measurement.

\section{Comparison of measuring techniques}

\section{1) Experimental group 1}

For the measurement of radiographic thickness (RT), wet soft tissue surfaces were covered with barium sulfate powder (SoloTop; Taejoon, Seoul, Korea, Fig. 2), a radiopaque material used as a gastrointestinal contrast medium, using a powder sprayer (Cerec Propellant; VITA Zahnfabrik, Bad Säckingen, Germany). Images were acquired with a CBCT scanner. Scanning parameters were 110 $\mathrm{kVp}, 24$ seconds, $5.7 \mathrm{~mA}$, a voxel size of $0.08 \mathrm{~mm}$, and a field of view of $5 \mathrm{~cm} \times 5 \mathrm{~cm}$. DICOM files were then assessed on viewer as follows. First, concentric circles of 9, 12 and $15 \mathrm{~mm}$ diameter were drawn from composite markers. Then, soft tissue thicknesses were measured at the intersection points between circle and soft tissue surface (Fig. 3). All gingival thicknesses were determined at the $0.01 \mathrm{~mm}$ level by the software. 


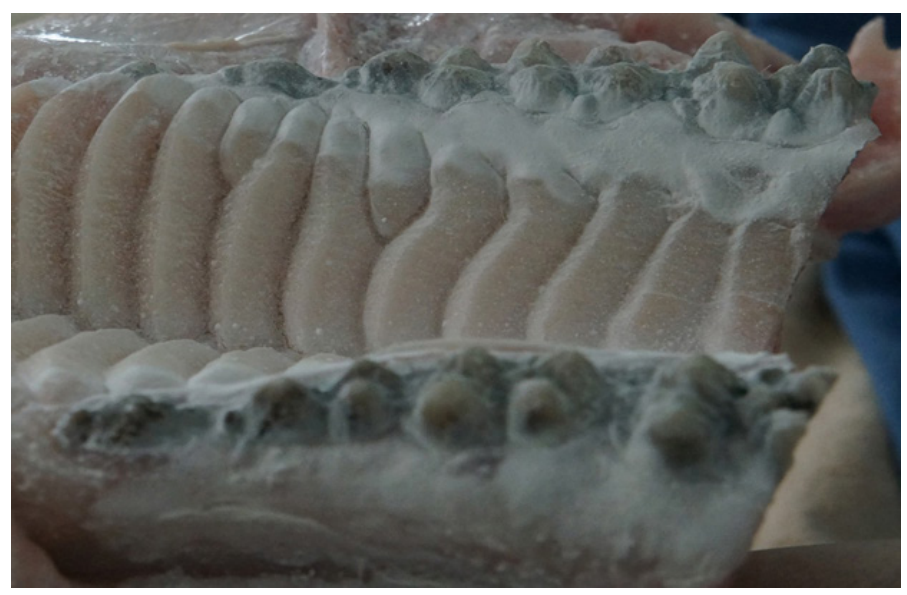

Fig. 2. Barium sulfate powder was sprayed on soft tissue surface.

Hae-Seok Lee et al. : Soft Tissue Measurement Method Using Radiopaque Material on Cone-beam Computed Tomography: An Ex Vivo Validation Study. Implantology 2018

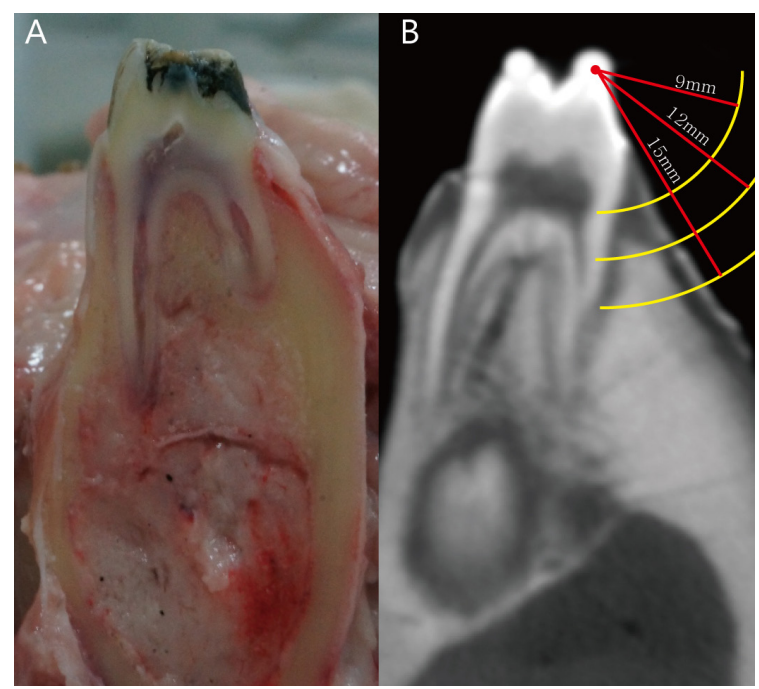

Fig. 3. Actual cross section of pig mandible (A) and CBCT image (B). Measurements were done $9 \mathrm{~mm}$, $12 \mathrm{~mm}, 15 \mathrm{~mm}$ apical from the markers.

Hae-Seok Lee et al. : Soft Tissue Measurement Method Using Radiopaque Material on Cone-beam Computed Tomography: An Ex Vivo Validation Study. Implantology 2018

\section{2) Experimental group 2}

A probes and rubber stop were used for the measurement of transgingival probing thickness (TPT). A periodontal probe (Williams PW, Hu-Friedy, Chicago, IL.) was inserted vertically into the soft tissue surface until resistance of the bone was felt. A rubber stop was placed in contact with the surface to facilitate the measurement of tissue thickness ${ }^{14}$. TPT was measured with digital calipers (Mitutuyo, Tokyo, Japan). All measurements were rounded to the nearest $0.01 \mathrm{~mm}$. 


\section{3) Control group}

For the measurement of actual thickness (AT), an incision was made onto each marked area. After flap elevation, actual soft tissue thickness was measured using the same method that was used for TPT. All measurements were rounded to the nearest $0.01 \mathrm{~mm}$.

\section{Statistical analysis}

Statistical analysis was consulted to independent statistician. SAS version 9.2 (SAS Institute, Cary, NC, USA) and MedCalc (MedCalc Software, Ostend, Belgium) version 12.7.0 were used for data analyses. The Kolmogorov - Smirnov test was used to determine whether variables were distributed normally. Correlations of RT and TPT values with AT values were examined using Pearson correlation coefficients. The distributions of variables were examined using dot plots. For reliability analysis, intraclass correlation coefficients (ICC) were calculated ${ }^{15}$ for AT, TPT, and RT. ICC values $>0.75$ were considered to have excellent reliability ${ }^{16}$. To graphically examine the degree of agreement between radiographic and actual measurements, a Bland - Altman plot was constructed, and limits of agreement were calculated for the outcome measure ${ }^{17} . P$-values $<0.05$ were considered to be statistically significant.

\section{Results}

\section{Intra-observer variability}

The paired t-test revealed no significant difference between the first and second readings. Also, correlation of the two measurements was significant (Pearson correlation coefficient on parameter $1=$ $0.99 ; P<0.01$, parameter $2=0.96 ; P<0.01$, parameter $3=0.97 ; P<0.01)$. The intra - observer variability and correlation coefficient were comparable to previous studies ${ }^{18}$.

\section{Comparison of measuring techniques}

All variables were distributed normally. Mean AT was $1.568 \pm 0.64 \mathrm{~mm}$, mean TPT was $1.759 \pm$ $0.74 \mathrm{~mm}$, and mean RT was $1.654 \pm 0.664 \mathrm{~mm}$ (Table 1). Coefficients of correlation between TPT and AT, and between RT and AT, were 0.810 and 0.892 , respectively (Table 2).

The ICC for TPT vs. AT was 0.87 (95\% confidence interval [CI], $0.79-0.92 ; p<0.0001)$, and that for RT vs. AT was 0.939 (95\% CI, $0.9-0.963 ; p<0.0001)$. The two ICC values differed significantly (Table 3 ).

Figure 4 is a Bland - Altman plot illustrating the degree of agreement between AT and RT values. The 
Table 1. Mean value and standard deviation for clinical measurements $(\mathrm{mm})(n=66)$

\begin{tabular}{cc} 
Variables & Mean \pm SD \\
Actual Thickness & $1.568 \pm 0.64$ \\
Transgingival probing Thickness & $1.759 \pm 0.74$ \\
Radiographic Thickness & $1.654 \pm 0.664$ \\
\hline
\end{tabular}

SD: standard deviation.

Hae-Seok Lee et al. : Soft Tissue Measurement Method Using Radiopaque Material on Cone-beam Computed Tomography: An Ex Vivo Validation Study. Implantology 2018

Table 2. Correlation coefficients with actual thickness and correlation comparison

\begin{tabular}{cccc} 
& TPT:AT & RT:AT & Correlation comparison $(P$-value $)$ \\
$r$ & 0.80951 & 0.89217 & 0.0103 \\
\hline
\end{tabular}

TPT: Transgingival probing Thickness; AT: Actual Thickness; RT: Radiographic Thickness; $r$ : Pearson's correlation coefficient.

Hae-Seok Lee et al. : Soft Tissue Measurement Method Using Radiopaque Material on Cone-beam Computed Tomography: An Ex Vivo Validation Study. Implantology 2018

Table 3. The intraclass correlation (ICC) with AT and ICC comparison

\begin{tabular}{cccccc} 
Variables & \multicolumn{2}{c}{ TPT } & \multicolumn{2}{c}{ RT } & $\begin{array}{c}\text { ICC comparison } \\
(P \text {-value })\end{array}$ \\
\cline { 2 - 5 } ICC & $0.87(0.788-0.92)$ & $<.0001$ & $0.939(0.9-0.963)$ & $<.0001$ & 0.0249 \\
\hline
\end{tabular}

CI: confidence interval.

TPT: Transgingival probing Thickness; AT: Actual Thickness; RT: Radiographic Thickness; $r$ : Pearson's correlation coefficient.

Hae-Seok Lee et al. : Soft Tissue Measurement Method Using Radiopaque Material on Cone-beam Computed Tomography: An Ex Vivo Validation Study. Implantology 2018

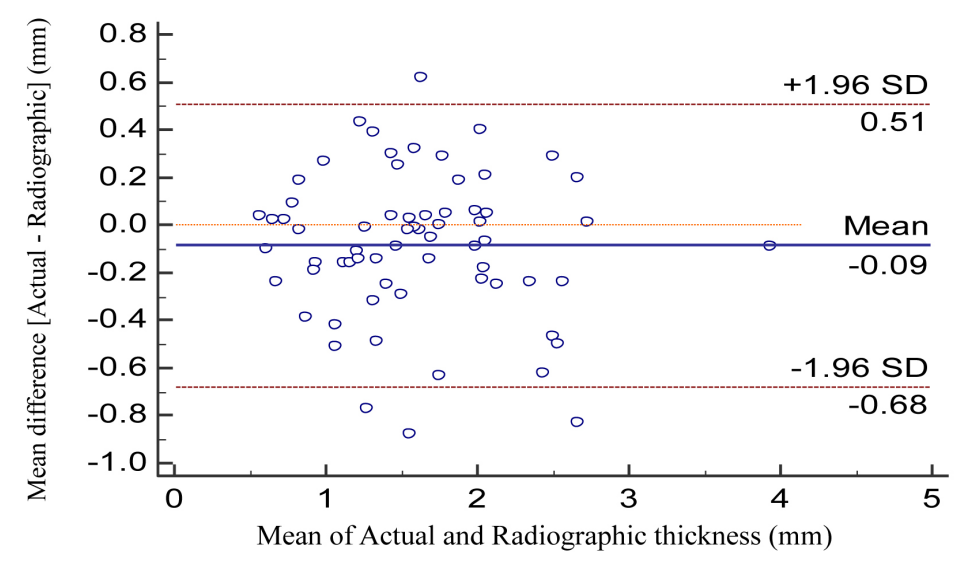

Fig. 4. Bland-Altman plots portraying the agreement between actual and radiographic measurements for soft tissue thickness. The solid line indicates the mean difference between actual and radiographic measurements; dashed line shows the $95 \%$ limits of agreement.

Hae-Seok Lee et al. : Soft Tissue Measurement Method Using Radiopaque Material on Cone-beam Computed Tomography: An Ex Vivo Validation Study. Implantology 2018 
mean discrepancy between thicknesses was $-0.09 \mathrm{~mm}$. The $95 \%$ limit of agreement for thickness was 0.51 to $-0.68 \mathrm{~mm}$.

\section{IV . Discussion}

The purpose of the present study was to validate a simple and noninvasive method of assessing the dimensions of soft tissues by CBCT. Strong significant correlations of AT with RT and TPT values were found, indicating that both methods are valid for the assessment of mucogingival soft tissue thickness. However, statistically significant difference was noted comparing coefficient values, indicating that correlation of RT and AT was stronger than that of TPT and AT. Although mean difference between RT and TPT might be minimal (mean difference $0.1 \mathrm{~mm}$ ) significant difference in correlation with AT suggests that RT might reflect AT better than TPT.

The radiographic measurement method showed good reliability, as indicated by the ICC. This result indicates that measurement error is small compared with the variability between AT and RT values. Comparable results were obtained for TPT. However, the significant difference between ICC values suggests that the radiographic measurements reflect actual gingival thickness than trans gingival probing, which is known to be the gold standard method. The narrow limit of agreement showed that the agreement of RT values was good.

Easy measurements performed with a periodontal probe, e.g., measurement of recession of gingiva, are fast and commonly part of routine diagnostic examination, but they provide limited three-dimensional information. The same applies to the ultrasonic method of soft tissue thickness measurement, which does not provide an overview of the periodontal structures or relationship ${ }^{19}$.

For the measurement of soft tissue dimensions, trans-gingival probing is commonly used method. ${ }^{1}$ However, this technique must be performed under local anesthesia, which might cause inadvertent volume change and discomfort to the patients. The use of radiopaque material enables the noninvasive measurement of soft tissue width and thickness, thereby allowing more accurate determination of the clinical prognosis. In addition, this method makes it possible to demarcate overlapped tissue area, such as buccal gingiva and cheek. However, possible drawbacks of using radiopaque material exists, such as additional cost for using radiopaque material, blurring of the image, and allergic reaction. These drawbacks should be dealt in depth for the possible routine clinical usage.

A previous study proposed a novel method based on CBCT technology called soft tissue CBCT to determine the relationship of the structures of the dento-gingival unit ${ }^{9}$. This method is a more suitable 
tool for the acquisition of an anatomic overview, and a more painless method to obtain images of the teeth and surrounding periodontal structures, compared with trans-gingival probing. However, verification of the method was not reported, and soft tissue dimensions can be underestimated considerably, depending on scan settings and tissue thickness ${ }^{6}$. Nevertheless, CBCT is part of the standard protocol for diagnosis and treatment planning in difficult cases with special questions. As correlation between bone and soft tissue is becoming important topic, this technique could play an increasingly significant role in dentistry as it is developed further.

In this study, we verified a method that utilizes the advantages of CBCT while overcoming disadvantages of this modality for the metric assessment of soft tissue dimensions around teeth and implants. Although additional research is needed to determine the proper concentration of contrast medium, the findings of this study suggest an opportunity to increase the usefulness for CBCT.

\section{Conclusion}

In conclusion, the results of the present study suggest that soft tissue measurement with CBCT and radiopaque material could be a reliable method, compared to the trans-gingival probing measurement method, with good validity and reproducibility.

\section{Acknowledgements}

This study was supported by a faculty research grant of Yonsei University College of Dentistry for 2015(6-2015-0121).

\section{References}

1. Lee DW, Kim CK, Park KH, et al. Non-invasive method to measure the length of soft tissue from the top of the papilla to the crestal bone. J Periodontol. 2005; 76: 1311-1314.

2. Lee DW, Park KH, Moon IS. Dimension of interproximal soft tissue between adjacent implants in two distinctive implant systems. J Periodontol. 2006; 77: 1080-1084.

3. Lee DW, Huh JK, Park KH, et al. Comparison of interproximal soft tissue height for single implants and contra-lateral natural teeth. Clin Oral Implants Res. 2009; 20: 1320-1325.

4. Claffey N, Shanley D. Relationship of gingival thickness and bleeding to loss of probing attachment in shallow sites following nonsurgical periodontal therapy. J Clin Periodontol. 1986; 13: 654-657.

5. Foushee DG, Moriarty JD, Simpson DM. Effects of Mandibular Orthognathic Treatment on 
Mucogingival Tissues. J Periodontol. 1985; 56: 727-733.

6. Ronay V, Sahrmann P, Bindl A, et al. Current status and perspectives of mucogingival soft tissue measurement methods. J Esthet Dent. 2011; 23: 146-156.

7. Scarfe WC, Farman AG, Sukovic P. Clinical applications of cone-beam computed tomography in dental practice. J Can Dent Assoc. 2006; 72: 75-80.

8. Guerrero ME, Jacobs R, Loubele M, et al. State-of-the-art on cone beam CT imaging for preoperative planning of implant placement. Clin Oral Investig. 2006; 10: 1-7.

9. Januario AL, Barriviera M, Duarte WR. Soft Tissue Cone-Beam Computed Tomography: A Novel Method for the Measurement of Gingival Tissue and the Dimensions of the Dentogingival Unit. J Esthet Restor Dent. 2008; 20: 366-373.

10. Barriviera M, Duarte WR, Januário AL, et al. Anew method to assess and measure palatal masticatory mucosa by cone-beam computerized tomography. J Clin Periodontol. 2009; 36: 564-568.

11. Cao J, Hu WJ, Zhang $\mathrm{H}$, et al. A novel technique for measurement of dentogingival tissue by cone beam computed tomography. Oral Surg Oral Med Oral Pathol Oral Radiol. 2015; 119: 82-87.

12. Silva JNN, Andrade PF, Sotto-Maior BS, et al. Influence of lip retraction on the cone beam computed tomography assessment of bone and gingival tissues of the anterior maxilla. Oral Surg Oral Med Oral Pathol Oral Radiol. 2017; 123: 714-720.

13. Ueno D, Sato J, Igarashi C, et al. Accuracy of oral mucosal thickness measurements using spiral computed tomography. J Periodontol. 2011; 82: 829-836.

14. Wara-aswapati N, Pitiphat W, Chandrapho N, et al. Thickness of palatal masticatory mucosa associated with age. J Periodontol. 2001; 72: 1407-1412.

15. Shrout PE, Fleiss JL. Intraclass correlations: uses in assessing rater reliability. Psychol Bull. 1979; 86: 420.

16. Fleiss JL. Reliability of measurement. In: Fleiss JL, The Design and Analysis of Clinical Experiments. 1st ed. New York, NY: John Wiley \& Sons; 1986. p.1-32.

17. Bland JM, Altman DG. Measuring agreement in method comparison studies. Stat Methods Med Res 1999; 8: 135-160.

18. Webber RL, Ruttimann UE, Heaven TJ. Calibration errors in digital subtraction radiography. J Periodontal Res. 1990; 25: 268-275.

19. Eger T, Müller HP, Heinecke A. Ultrasonic determination of gingival thickness. J Clin Periodontol 1996; 23: 839-845. 
